\title{
The Study of Thermal Properties of GaAs/AlGaAs Quantum Cascade Lasers
}

\author{
E. Pruszyńska-Karbownik, P. Karbownik, A. Szerling, K. Kosiel and M. Bugajski \\ Institute of Electron Technology, al. Lotników 32/46, 02-668 Warsaw, Poland
}

\begin{abstract}
Temperature change in quantum cascade laser can be estimated by studying the device resistance change. Using this method we compared quantum cascade laser structure mounted on diamond heat spreader and without heat spreader. We have shown that the use of heat spreader reduces temperature increase even by $40 \%$.
\end{abstract}

PACS numbers: 42.55.Px, 85.60.-q, 85.35.Be, 72.80.Ey, 85.60.-q

\section{Introduction}

The quantum cascade lasers (QCLs) are unipolar semiconductor light sources based on intersubband transition and on tunneling. Active region of the QCL is formed by alternating coupled quantum wells and superlattice regions (injectors). The whole active region has thickness of several micrometers and contains hundreds of nanometer semiconductor layers. Thermal conductivity of such structure can be even ten times smaller than thermal conductivity of bulk material $[1,2]$. For that reason thermal management is crucial issue in designing, fabrication and testing of quantum cascade lasers.

Several methods to investigate thermal properties of QCLs have been proposed recently. There are based on studies of emitted wavelength [3] or on photoluminescence [4-6]. Using those methods temperature of active region can be estimated. Contrary to photoluminescence method, wavelength method can measure rapid temperature changes during pulse excitation. Disadvantage of that method is a fact that only structures whit good optical properties can be studied. Therefore it cannot be used as a diagnostic tool.

In this paper we propose an alternative method of QCL temperature estimation based on electrical properties study. Measured temperature is the temperature of the whole structure. This method allows for measurement of rapid temperature changes and does not need good optical properties of the laser. Another advantage is the fact that no sophisticated experimental setup is needed.

\section{Experiment}

The basic of our temperature measurement method is changing of device resistance with the temperature. For determining of this dependence we measured the voltage at the beginning of the impulse for fixed current at several external temperatures. Then, the changes of voltage and current during impulse for fixed external temperature were measured. Using the temperature-resistance dependence determined for each structure we estimate temperature changes during excitation.

In our experimental setup the QCL was supplied by power supply with current control, whose rise time was 40 ns. This time was not counted as the excitation time. The structure temperature was adjusted by a Peltier device. The voltage and current wave forms were measured by a digital oscilloscope.

In the experiment we use AlGaAs/GaAs QCL structures MBE grown on GaAs substrate. Full details about epitaxy and processing are contained in Ref. [7]. Emitting wavelength was about $9 \mu \mathrm{m}$. In this paper two structures are compared. One was soldered with AuSn onto diamond heat spreader onto Au-plated copper mount (sample A) and second soldered with AuSn onto copper mount without heat spreader (sample B).

\section{Results and discussion}

The exemplary dependence of the electrical resistance as a function of the temperature which was experimentally determined for sample $\mathrm{A}$ is shown in Fig. 1. We have fitted it with linear dependence.

Also exemplary voltage and current wave forms for sample A is presented in Fig. 2. An appreciable voltage change during the impulses, which is concerned with resistance change, is noticeable.

To compare temperature changes we have supplied both samples with the same instantaneous electrical power, which was $41 \mathrm{~W}$. The external ambient temperature was $293 \mathrm{~K}$. The measured temperature changes for both samples are presented in Fig. 3 .

After $500 \mathrm{~ns}$ of excitation the temperature of sample A increased by $(60 \pm 5) \mathrm{K}$ and the temperature of sample $\mathrm{B}$ increased by $(77 \pm 8) \mathrm{K}$. These temperature increase for $900 \mathrm{~ns}$ impulses was respectively $(81 \pm 5) \mathrm{K}$ for sample A and $(147 \pm 10) \mathrm{K}$ for sample B. 


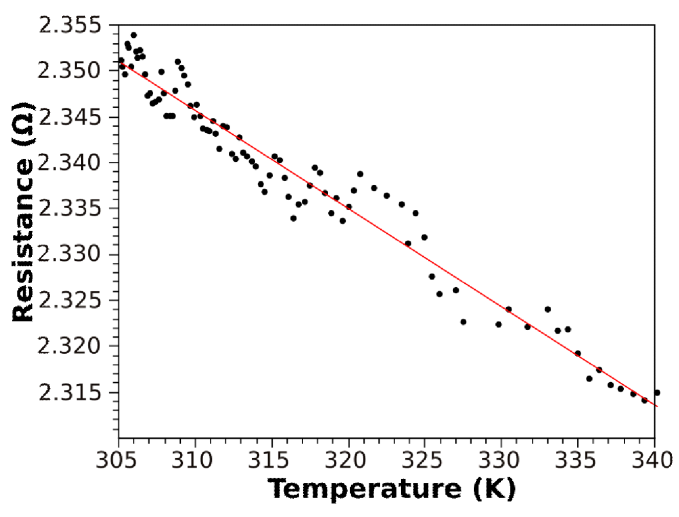

Fig. 1. Electrical resistance as a function of temperature for sample A.

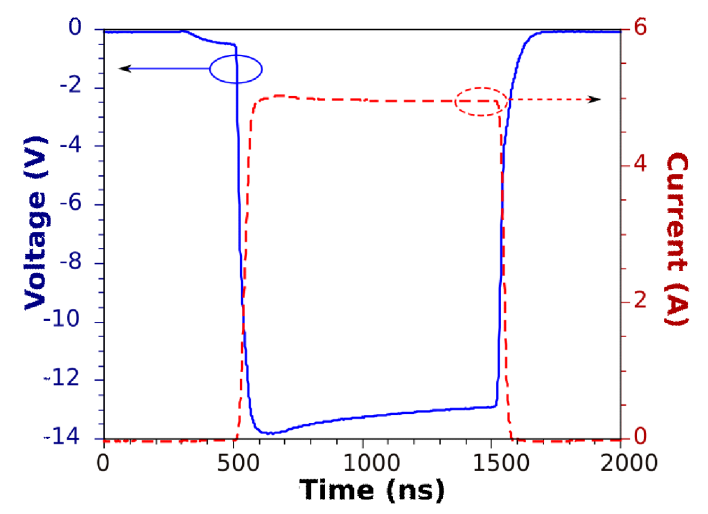

Fig. 2. Voltage and current wave forms for sample A.

These values can not be directly compared with results from other published papers because there were measured only active region temperatures, and there were used different QCL structures. However value obtained by Tober [3] for $36 \mathrm{~W}$ of instantaneous electrical power and for $500 \mathrm{~ns}$ of excitation was about $50 \mathrm{~K}$ and has the same order of magnitude as our result. The measured values

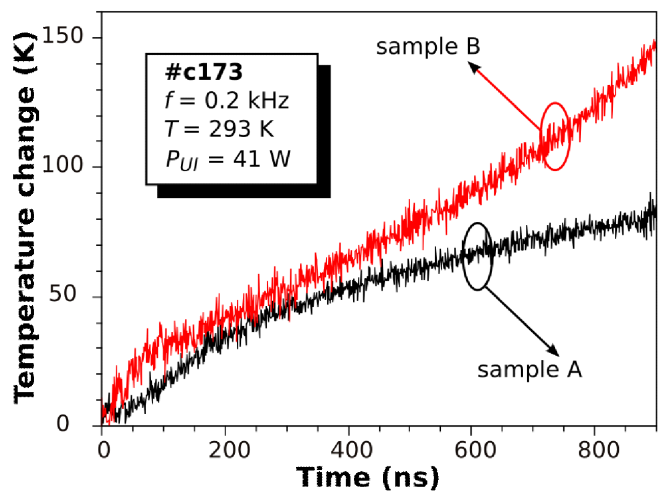

Fig. 3. Change of the device temperature during excitation as a function of time for samples A and B. of the temperature increase are also in agreement with theoretical predictions [8].

\section{Summary}

In this paper we have shown that studying of resistance changes is a simple and useful high-speed technique for device temperature estimation. We have compared QCL structures from the same wafer with the same processing but of different type of mounting. We have shown that the use of diamond heat spreader can reduce temperature growth by over $40 \%$ for $900 \mathrm{~ns}$ impulses. Mounting without heat spreader can be used only for lasers which work with relatively short impulses (to $200 \mathrm{~ns}$ ).

\section{Acknowledgments}

The authors would like to acknowledge L. Ornoch and M. Wasiak for helpful discussions and I. Makarowa for chip mounting. The work was partially supported by grant PBZ-MIN-02/I/2007.

\section{References}

[1] W.S. Capinski, M. Cardona, D.S. Katzer, H.J. Maris, K. Ploog, T. Ruf, Physica B 263-264 530 (1999).

[2] J. Piprek, T. Troger, B. Schroter, J. Kolodzey, C.S. Ih, IEEE Photon. Technol. Lett. 10, 81 (1998).

[3] R.L. Tober, J. Appl. Phys. 101, 004507 (2007).

[4] V. Spagnolo, M. Troccoli, G. Scamarcio, C. Gmachl, F. Capasso, A. Tredicucci, A.M. Sergent, A.L. Hutchinson, D.L. Sivco, A.Y. Cho, Appl. Phys. Lett. 78, 2095 (2001).

[5] A.J. Borak, C.C. Phillips, C. Sirtori, Appl. Phys. Lett. 82, 4020 (2003).

[6] M.S. Vitiello, G. Scamarcio, V. Spagnolo, Appl. Phys. Lett. 92, 101116 (2008).

[7] K. Kosiel, M. Bugajski, A. Szerling, J. Kubacka-Traczyk, P. Karbownik, E. Pruszyńska-Karbownik, J. Muszalski, A. Łaszcz, P. Romanowski, M. Wasiak, W. Nakwaski, I. Makarowa, P. Perlin, Photonics Lett. Poland 1, 16 (2009).

[8] M. Wasiak, R.P. Sarzała, W. Nakwaski, in: VIII Krajowa Konferencja Elektroniki, Dartówko Wschodnie (Poland) 2009. 